\title{
Soft Mode Metal-Linker Dynamics in Carboxylate MOFs
}

\author{
Anastasia B. Andreeva, Lihaokun Chen, Khoa N. Le, \\ Michael E. Kellman, Christopher H. Hendon*, Carl K. Brozek* \\ Department of Chemistry and Biochemistry, Materials Science Institute, \\ University of Oregon, Eugene, OR 97405 \\ Email: cbrozek@uoregon.edu
}

\begin{abstract}
Through comprehensive analysis of carboxylate-based metal-organic frameworks (MOFs), we present general evidence for dynamic metal-linker bonding that challenges the common perception of MOF structures being static. Structural dynamics in MOFs, however, typically refers to the "breathing" behavior of pore cavities and the transient binding of guest molecules, but dynamic bonding would explain important MOF phenomena in catalysis, postsynthetic exchange, negative thermal expansion, and crystal growth. Here, we demonstrate through use of variable-temperature diffuse reflectance infrared Fourier transform spectroscopy (VTDRIFTS) aided by ab initio plane wave density function theory, that similar evidence for melting behavior in zeolitic imidazolate frameworks (ZIFs) can be observed for carboxylate MOFs by monitoring the red-shifts of carboxylate stretches coupled to anharmonic metal-carboxylate oscillators. To demonstrate the generality of these findings, we investigate a wide class of carboxylate MOFs that includes iconic examples with diverse structures and metal-linker chemistry. As the very vibrations invoked in ZIF melting, but heretofore unobserved for carboxylate MOFs, these metal-linker dynamics resemble the ubiquitous soft modes that trigger important phase transitions in diverse classes of materials, while offering a fundamentally new perspective for the design of next-generation metal-organic materials.
\end{abstract}

\section{Introduction}

Important material phenomena often depend on dynamic distortions to solid lattices, such as ion diffusion through solid electrolytes ${ }^{1}$ and surface reconstruction of heterogeneous catalysts. ${ }^{2}$ In particular, certain lattice vibrations cause such extreme distortions to equilibrium geometries that they trigger structural phase transitions responsible for wide-ranging functional properties, ${ }^{3}$ including ferroelectricity, ${ }^{4-7}$ metal-insulator transitions, ${ }^{8}$ exciton condensation, ${ }^{9,10}$ and multiferroics. ${ }^{11}$ Monitoring these phonon modes as a function of temperature reveals that the peak positions redshift and intensities diminish near the phase change critical temperature due to the vibrations transferring energy into the structural reordering process through anharmonic coupling 
to other phonons. ${ }^{12}$ Because these vibrations dissipate energy, they are termed "soft modes". Analysis of such vibrations, and their coupling to magnetic, vibrational, electronic, and other degrees of freedom, offers a microscopic basis for predicting and controlling phase transitions. ${ }^{13}$ Understanding the relationship of lattice dynamics to phase transitions has guided the design of new types of materials, such as metastable phases, for devices with specifically enhanced physical properties. Recently, metal-linker dynamics in the porous coordination polymers known as metalorganic frameworks (MOFs) have been invoked to describe melting mechanisms of MOF liquids ${ }^{14-18}$ and glasses. ${ }^{19-26}$ These disordered MOF materials attract considerable attention by opening possibilities in the design of porous materials for applications ranging from gas storage to ion exchange membranes, but the metal-ligand dynamics associated with their melting transitions challenge the common conception of MOFs as static structures. Although metal-linker lability helps explain melting and other important behavior, evidence for such dynamic coordination chemistry has only been observed indirectly for carboxylate MOFs. ${ }^{27-29}$ Direct measurement of metal-linker lability has been possible for certain ZIFs, however, requiring specialized hightemperature synchrotron techniques. ${ }^{14,24}$ The intensifying research into dynamic metal-organic materials would benefit, therefore, from routine methods that probe metal-linker lability, especially for carboxylate MOFs, which constitute the overwhelming majority of MOFs.

Although reversible metal-linker coordination is thought to drive MOF crystallization, such dynamic bonding is not commonly thought to occur once the MOF is formed. Dynamic metallinker bonding is well-documented in analogous one-dimensional coordination polymers, which comprise an important subset of materials termed "dynamic", "adaptable", "stimuli-responsive", or "self-healing covalent networks" ${ }^{30-34}$ For example, formation constants $\left(\log K_{f}, K_{f}=[\right.$ bound metal linkers]/[unbound metal linkers]) of metal-pyridy ${ }^{35}$ and metal-carbene ${ }^{36}$ polymers have 
been measured to be as small as 3 to 4 , implying that "unbound" states constitute a significant portion of the polymer linkages. Interestingly, formation constants of molecular metal-carboxylate complexes akin to MOF coordination moieties, e.g., zinc-benzoate, range from only 0.1 to $1.5 .{ }^{37}$ Structural dynamics in MOFs, instead, conventionally refers to the pressure-induced "breathing" behavior of pore cavities ${ }^{38-41}$ or to the transient binding of guest molecules, ${ }^{27,42-46}$ but labile metallinker bonding would explain important phenomena, such as the ability of MOFs to undergo postsynthetic exchange, ${ }^{47-53}$ exhibit negative thermal expansion, ${ }^{54-58}$ perform catalysis at seemingly saturated metal centers, ${ }^{59}$ and readily grow as bulk crystals. Evidence for metal-linker dynamics could, therefore, guide deliberate control over these important functions, while inspiring the design of porous materials with stimuli-responsive, metastable, self-healing, and other adaptable behavior. By challenging the common conception of carboxylate MOFs as static structures, evidence for their labile metal-linker bonding will enable their design in applications that leverage structural dynamics.

Here, we demonstrate variable-temperature diffuse reflectance infrared Fourier transform spectroscopy (VT-DRIFTS) as a convenient method for probing MOF metal-linker dynamics. We observe that at higher temperatures, carboxylate stretches red-shift for a general collection of representative carboxylate-based MOF materials, which we ascribe to thermal population of MOF conformations with "loose" metal-carboxylate linkages in equilibrium with a decreased population of "tight" metal-linker states. Importantly, the $\mathrm{C}-\mathrm{O}$ stretches provide a convenient spectroscopic handle for metal-linker bonding dynamics, due to the coupling of the readily observable $\mathrm{C}-\mathrm{O}$ modes to the more elusive $\mathrm{M}-\mathrm{O}$ modes (Scheme 1A). A similar approach has been employed previously, where analysis of linker, rather than metal-linker vibrational modes, provided evidence for dynamic bonding in self-healing metal-pyridyl one-dimensional coordination polymers. ${ }^{60}$ 
Variable-temperature Raman spectroscopy evidenced red-shifting pyridyl stretches at higher temperatures that were attributed to dynamic structural rearrangements. ${ }^{60}$ On the other hand, evidence for the melting mechanism of ZIFs rests on analysis of red-shifting zinc-imidazolate bond stretches, requiring specialized variable-temperature terahertz (THz)/Far-IR synchrotron techniques, due to the low-energy spectral range. ${ }^{24}$ In these reports, consistent with analysis of other dynamic and phase-change systems, the red-shifting behavior was attributed to thermal population of the unevenly spaced vibrational excited states of an anharmonic Zn-imidazolate oscillator. ${ }^{61}$ Because the states become more closely spaced at higher energies, the apparent vibrational mode shifts to lower wavenumbers. With greater population of higher energy states, the vibrational amplitudes also increase until they reach a critical ratio with respect to the interatomic metal-ligand spacing, termed the Lindemann ratio, ${ }^{62}$ causing the material to melt. Fundamentally, this behavior resembles the ability of soft modes to induce phase transitions through vibrational motion. Here, we demonstrate that similar evidence for melting behavior can be observed for carboxylate MOFs by monitoring the red-shifts of carboxylate stretches coupled to anharmonic metal-linker oscillators. We justify this strategy in terms of molecular orbital arguments and a simple two-state model of tight and loose metal-linker states in thermal equilibrium. The temperature-induced metal-linker dynamics evidenced in these prototypical MOFs resembles VT-DRIFTS studies of soft modes and phase changes in other classes of materials, including ZIFs, but had not been previously observed for ubiquitous carboxylate MOFs. Evidence for soft modes in carboxylate MOFs have been previously impeded by the difficulty of monitoring low-energy metal-linker vibrations directly, whereas the carboxylate stretches studied here provide a convenient alternative spectroscopic handle. To demonstrate the generality of these findings, we investigate a wide class of carboxylate MOFs that includes iconic examples with 
diverse structures and metal-linker chemistry. The broad applicability of these results offers a fundamentally different perception of MOFs. In addition to MOFs, VT-DRIFTS offers a powerful method for probing the stability, dynamics, and structure of the wide class of organic-inorganic materials.

Scheme 1: Molecular orbital (MO) description of MOF metal-carboxylate interactions. (A) Schematic representation of $\mathrm{C}-\mathrm{O}$ and $\mathrm{M}-\mathrm{O}$ coupled anharmonic oscillators in metal-carboxylate complexes. (B) MO diagram of carbonyl bond in a carboxylate group.

A

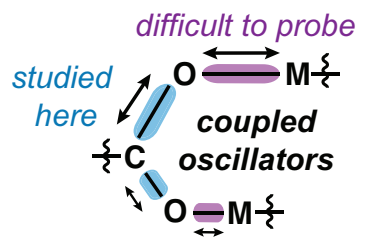

\section{Results and Discussion}

B $\quad \sigma^{*}$

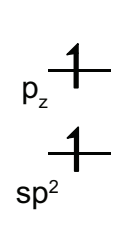

C
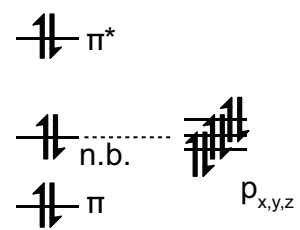

0

Molecular orbital (MO) theory provides a straightforward explanation for why carboxylate stretches might redshift with weaker metal-linker interactions. Scheme 1B illustrates a simplified MO diagram for an individual $\mathrm{C}-\mathrm{O}$ bond of a carboxylate group. Because the highest energy electrons reside in orbitals that are anti/non-bonding with respect to the $\mathrm{C}-\mathrm{O}$ bond, they act as the lone pair involved in dative interactions with metal ions. Accordingly, stronger $\mathrm{M}-\mathrm{O}$ interactions would also enhance $\mathrm{C}-\mathrm{O}$ bonding by distributing the anti-bonding density away from the $\mathrm{C}-\mathrm{O}$ bond vector. Therefore, strong M-O interactions should cause blueshifts to carboxylate stretches, and weak M-O interactions should cause redshifts. Indeed, strongly ionic carboxylate complexes, such as sodium benzoate, show the highest-energy carboxylate stretching modes. For similar reasons, interactions between Lewis acidic metal ions and carbon monoxide induce blueshifts to $\mathrm{C}-\mathrm{O}$ stretching frequencies. Hence, we hypothesize that if MOF metal-linker bonds exist in equilibrium between "tight" and "loose" states, then high temperatures would produce redshifts 
by shifting the equilibrium toward weaker $\mathrm{C}-\mathrm{O}$ interactions. Second, if observed carboxylate bands arise from overlapping spectral components from tight and loose species, then we expect band widths to maximize at temperatures where these species exist in equal mixtures and to minimize at temperatures dominated by a single species.
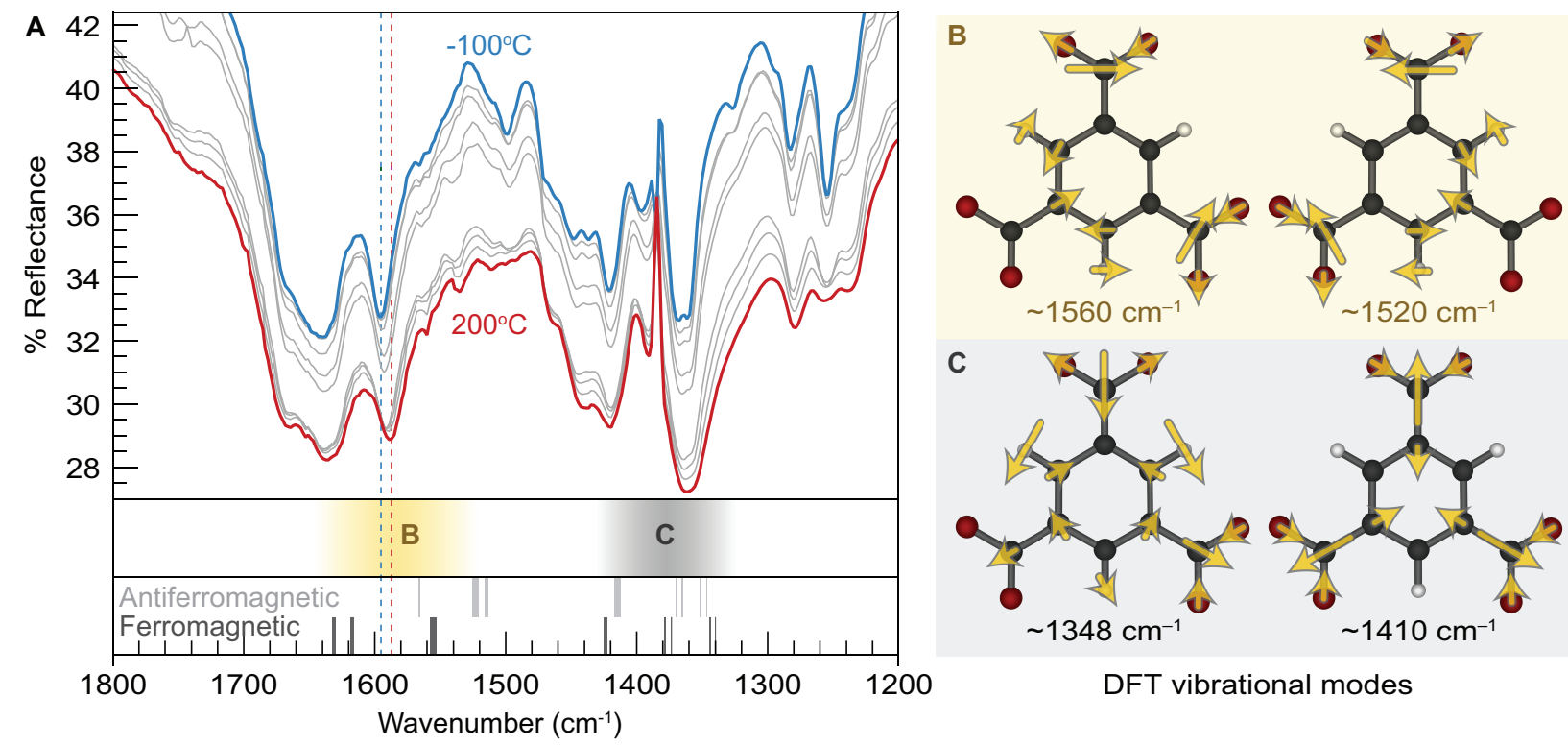

DFT vibrational modes

Figure 1: Variable-temperature diffuse reflectance infrared Fourier transform spectra (VTDRIFTS) and computed phonon modes of HKUST-1. (A) (Top) Experimental spectra collected between $-100{ }^{\circ} \mathrm{C}$ and $200{ }^{\circ} \mathrm{C}$ under dynamic vacuum. (Bottom) Simulated peak positions of phonon modes with carboxylate character denoted by color intensity and labeled according to modes show in panel B. (B) Representation of "asymmetric" and (C) "symmetric" carboxylatebased phonon modes projected at the $\Gamma q$-point.

The material HKUST-1 $\left(\mathrm{Cu}_{3}(1,3,5 \text {-benzenetricarboxylate })_{2}\right)$ was chosen as an initial target for VT-DRIFTS as many seminal studies of MOFs were first demonstrated with this material. Figure 1A plots spectra collected at $50{ }^{\circ} \mathrm{C}$ intervals starting from room temperature, then warmed to $200{ }^{\circ} \mathrm{C}$, and then cooled to $-100{ }^{\circ} \mathrm{C}$. Surprisingly, several bands, in addition to the expected asymmetric and symmetric carboxylate stretches, appear to redshift with increased temperature. Therefore, we employed density functional perturbation theory for proper assignment of all bands in Figure 2A. As the Cu paddlewheel dimer in HKUST-1 displays antiferromagnetic ordering 

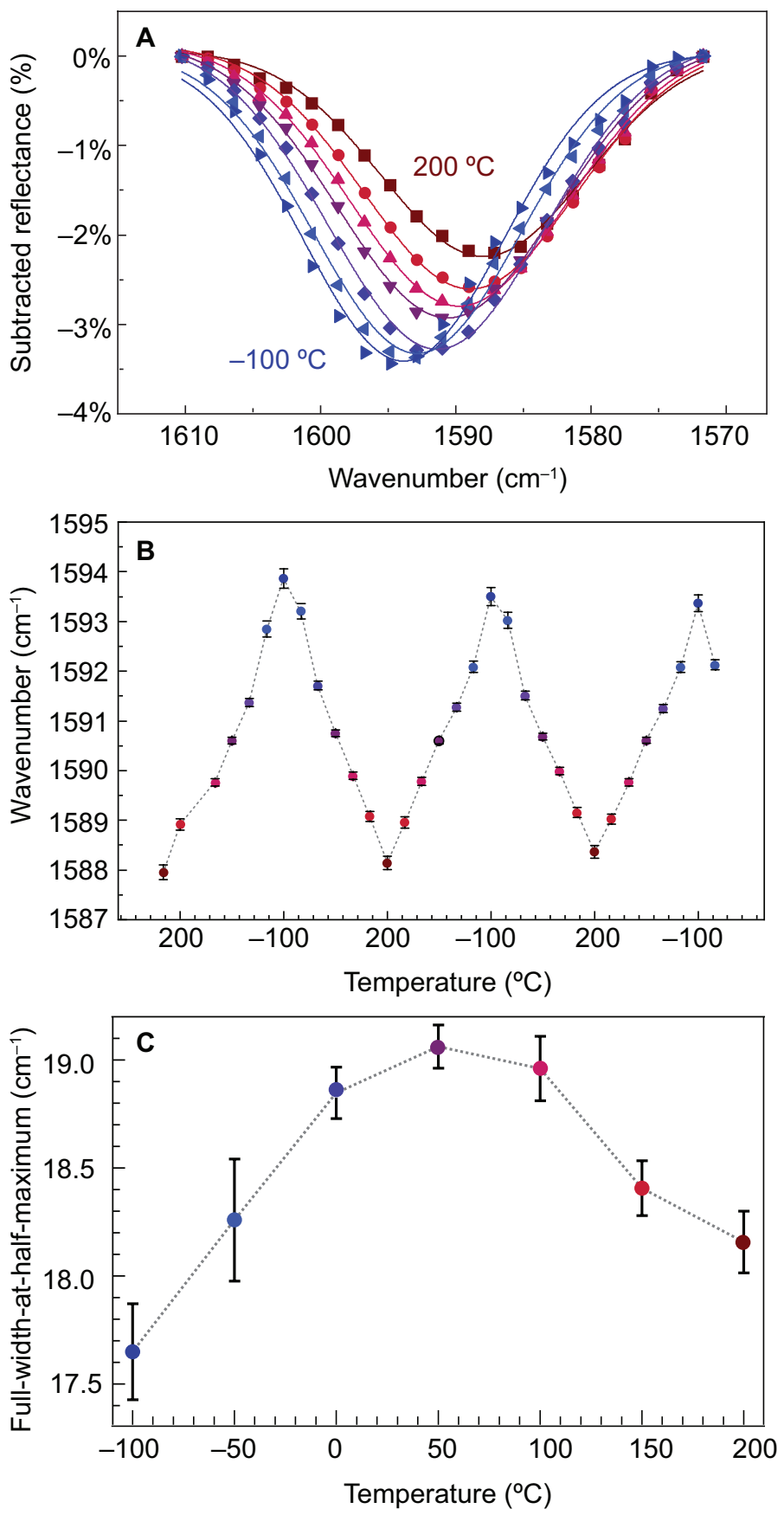

Figure 2: Temperature-dependent spectral data for the HKUST-1 asymmetric carboxylate vibrational mode. (A) Baseline-subtracted reflectance spectra, with experimental data shown as markers and Gaussian fits shown as solid lines. (B) Peak positions plotted against temperature across three continuous temperature ramp cycles, with error bars derived from Gaussian fits and data point colors corresponding to temperatures shown in panel A. (C) Full-width-at-halfmaximum (FWHM) values averaged over three temperature ramp cycles. Error denotes denote standard deviations from the averaged data. 
below $280 \mathrm{~K},{ }^{63}$ which is within the measured temperature regime, spectra were simulated with both antiferromagnetic (AMF) and ferromagnetic (FM) ordering, with the former showing excellent agreement with spectra collected below room temperature (Figure 1A, bottom). Notably, the bands unique to the AFM magnetic state help account for experimental features, such as those at 1499 and $1326 \mathrm{~cm}^{-1}$, that disappear above $300 \mathrm{~K}$. To aid in band assignment, vibrational normal modes were projected at the $\Gamma q$-point and represented as molecular diagrams (Figure 1B), with arrows denoting vibrational directions and oscillator intensities. Interestingly, all contain carboxylate character, suggesting that the temperature dependence of the data in Figure 1A arises from orbital interactions involving carboxylates specifically. Among these bands, the symmetric carboxylate stretch shows the most distinctive redshift. Consequently, we explicate the following analysis by focusing on this band in particular, although the symmetric and asymmetric carboxylate stretches of all MOFs considered here exhibit redshifts.

To highlight the apparent temperature dependence, Figure 2A plots the baseline-subtracted asymmetric carboxylate stretches collected between $-100{ }^{\circ} \mathrm{C}$ and $200{ }^{\circ} \mathrm{C}$. This band shifts by 5.8 $\mathrm{cm}^{-1}$ from a maximum frequency of $1594.8 \mathrm{~cm}^{-1}$ at $-100{ }^{\circ} \mathrm{C}$ to a minimum value of $1589.0 \mathrm{~cm}^{-1}$ at $200^{\circ} \mathrm{C}$. For closer inspection, Figure 3B shows the peak maxima versus temperature, cycled three times between $-100{ }^{\circ} \mathrm{C}$ and $200{ }^{\circ} \mathrm{C}$, with values obtained from Gaussian fits to each band. These data reveal a linear relationship between $-100{ }^{\circ} \mathrm{C}$ and $200{ }^{\circ} \mathrm{C}$ with a slope, $X_{a}$, of $-0.023 \mathrm{~cm}^{-1} / \mathrm{K}$, and confirm reversibility, as expected for a process in thermodynamic equilibrium. Furthermore, the symmetric stretch shifts between 1367.9 and $1362.1 \mathrm{~cm}^{-1}$ in this temperature regime (Figure S6-7), while the additional peaks around 1640 and $1400 \mathrm{~cm}^{-1}$ with partial carboxylate character also redshift. Interestingly, these plots resemble temperature-dependent vibrational studies of minerals, ZIFs, and other classes of materials that relate shifting peak maxima to interrelated 
concepts of phase changes, lattice instability, and structural dynamics. For example, the $v_{2}$ band of calcite, said to possess a "softer interatomic potential" than the other bands, exhibits a $X$ of $0.0049 \mathrm{~cm}^{-1} / \mathrm{K}$ near the critical temperature for phase transitions into calcium carbonate polymorphs. ${ }^{64}$ Redshifting vibrations have also been observed widely in other minerals, such as apatite, and are attributed to temperature-dependent anharmonic coupling between phonon modes ${ }^{65}$ Additionally, the zinc-imidazolate-based ZIF-UC-4 displays a $X$ of $-0.00292 \mathrm{~cm}^{-1} / \mathrm{K}$ in the crystalline phase, yet $X$ values of $-0.00597 \mathrm{~cm}^{-1} / \mathrm{K}$ and $-0.1377 \mathrm{~cm}^{-1} / \mathrm{K}$ in the glass and liquid phases, respectively. ${ }^{24}$ Taken together, these reports suggest that $X$ offers a measure of the susceptibility of bonds to distort in response to temperature. Larger slopes, therefore, represent weaker bonding. Importantly, the average full-width-at-half-maximum (FWHM) values increase from $-100{ }^{\circ} \mathrm{C}$ to a maximum around $50{ }^{\circ} \mathrm{C}$, followed by decreasing band width toward $200{ }^{\circ} \mathrm{C}$. These data strongly suggest the presence of multiple spectral components representing species in thermodynamic equilibrium.

To explore the generality of temperature-dependent MOF carboxylate stretches, a suite of representative MOF materials was investigated that offer a range of metal-carboxylate binding geometries and metal-linker bond strengths. These were chosen because seminal findings have been made with these examples: Mg-MOF-74 ( $\mathrm{Mg}_{2}$ (2,5-dihydroxyterephthalate)), Zn-MOF-74 $\left(\mathrm{Zn}_{2}(2,5\right.$-dihydroxyterephthalate) $\left.), \quad \mathrm{MOF}-5 \quad\left(\mathrm{Zn}_{4} \mathrm{O}(1,4-\text { benzenedicarboxylate })\right)_{3}\right), \quad$ MIL-125 $\left(\mathrm{Ti}_{8} \mathrm{O}_{8}(\mathrm{OH})_{4}(1,4 \text {-benzenedicarboxylate })_{6}\right)$, MIL-125- $\mathrm{NH}_{2}\left(\mathrm{Ti}_{8} \mathrm{O}_{8}(\mathrm{OH})_{4}(2 \text {-aminoterephthalate })_{6}\right)$, UiO-66 $\quad\left(\mathrm{Zr}_{6} \mathrm{O}_{6}(1,4-\text {-benzenedicarboxylate })_{6}\right), \quad \mathrm{MUV}-10(\mathrm{Ca}) \quad\left(\mathrm{Ti}_{3} \mathrm{Ca}_{3} \mathrm{O}_{2}(1,3,5-\right.$ benzenetricarboxylate) $)_{4}$, and $\mathrm{MUV}-10(\mathrm{Mn}) \quad\left(\mathrm{Ti}_{3} \mathrm{Mn}_{3} \mathrm{O}_{2}(1,3,5 \text {-benzenetricarboxylate })_{4}\right)$. All materials were subjected to identical measurement conditions and resulting spectra were analyzed by comparing to simulated spectra, as described for HKUST-1. Indeed, all MOFs exhibited clear 
red-shifts to vibrational modes upon heating from $-100{ }^{\circ} \mathrm{C}$ to $200{ }^{\circ} \mathrm{C}$, as summarized in Table 1 . As expected for a diverse collection of coordination environments and orbital interactions, the observed temperature-induced shifts vary significantly between MOF materials, even with isostructural analogs. Interestingly, the asymmetric stretch, typically ascribed to $\mathrm{C}=\mathrm{O}$ double bond character, exhibited greater sensitivity in certain materials, such as MOF-5, Mg-MOF-74, and ZnMOF-74, whereas the symmetric stretch, often associated with $\mathrm{C}-\mathrm{O}$ single bond character, showed greater sensitivity in others. According to the MO explanation, the linker-based stretching frequencies of MOFs with strong metal-linker bonds should show little if any temperature dependence. This interpretation helps explain the temperature-independent triazolate stretches observed in VT-DRIFTS studies of Zn-CFA-1. ${ }^{66}$ Interestingly, MIL-125, MIL-125-NH2, and UiO66, show temperature-dependent carboxylate shifts despite their notable structural stability. Yet, as expected, the observed redshifts slopes $X$ appear smallest among these examples. Because molecular metal carboxylate complexes feature similar orbital interactions to these MOFs, they should also exhibit such temperature dependence. Indeed, we observe from VT-DRIFTS measurements that sodium benzoate shows large shifts to both asymmetric and symmetric carboxylate stretches (Table 1).

Table 1: Summary of asymmetric $\left(X_{\mathrm{a}}\right)$ and symmetric $\left(X_{\mathrm{s}}\right)$ red-shift slopes of carboxylate materials studied here through VT-DRIFTS collected under dynamic vacuum.

\begin{tabular}{ccc}
\hline MOF Name & $\boldsymbol{X}_{\mathbf{a}}\left(\mathbf{c m}^{-\mathbf{1}} / \mathbf{K}\right)$ & $\boldsymbol{X}_{\mathbf{s}}\left(\mathbf{c m}^{-\mathbf{1}} / \mathbf{K}\right)$ \\
\hline \hline HKUST-1 & -0.021 & -0.020 \\
MUV-10 $(\mathrm{Ca})$ & -0.019 & -0.019 \\
MUV-10 $(\mathrm{Mn})$ & -0.015 & -0.041 \\
Mg-MOF-74 & -0.027 & -0.0087 \\
Zn-MOF-74 & -0.023 & -0.0042 \\
MOF-5 & -0.026 & -0.0095 \\
MIL-125 & -0.0093 & -0.019 \\
MIL-125-NH 2 & -0.036 & -0.066 \\
UiO-66 & -0.015 & -0.020 \\
\hline \hline Sodium benzoate & -0.015 & -0.024 \\
\hline
\end{tabular}




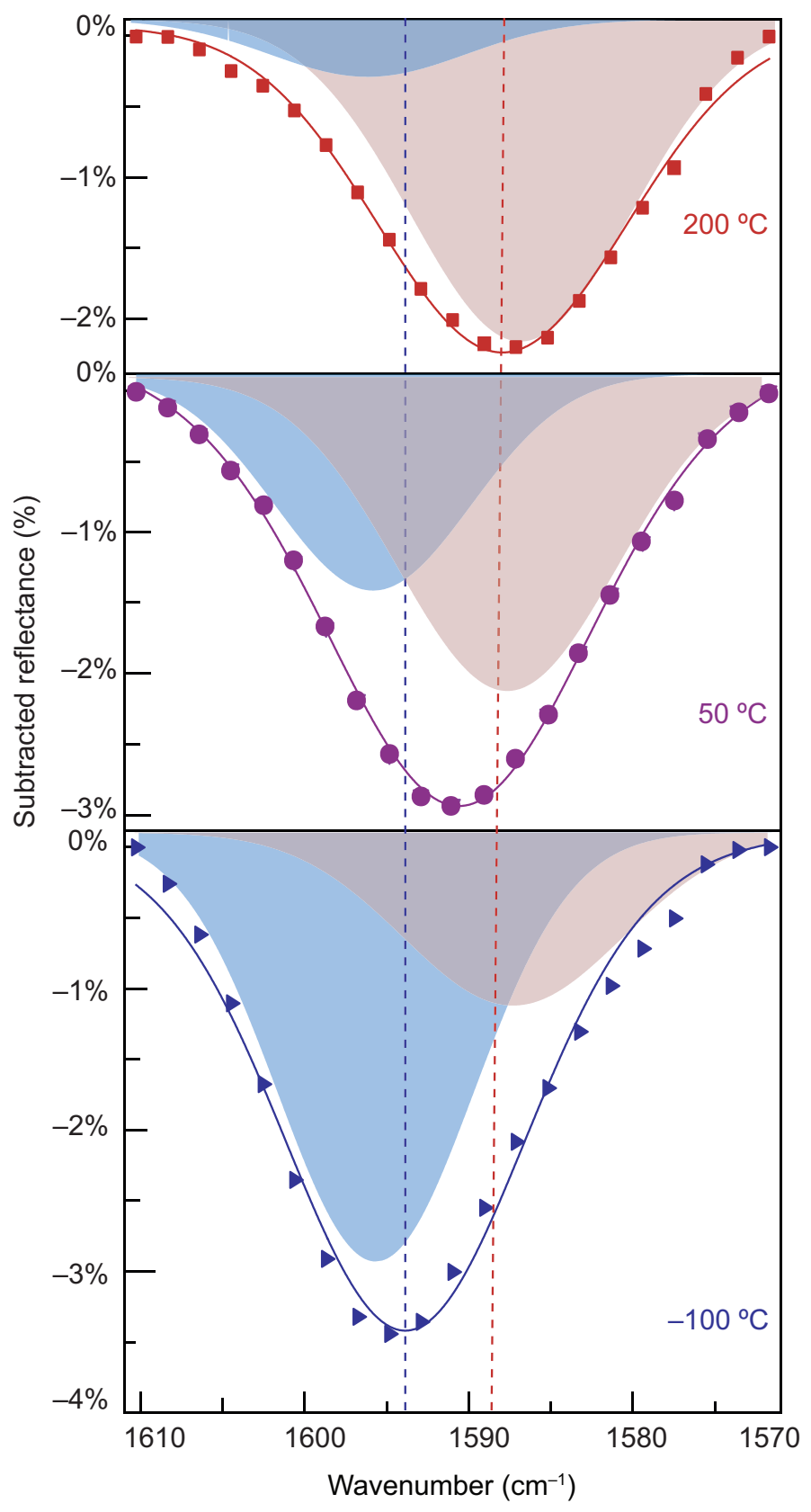

Figure 3: Global curve fittings for representative spectra of the HKUST-1 asymmetric carboxylate stretch. Spectral deconvolution assumed two components with constant peak maxima but variable total areas. Fits produced a species centered at $1596 \mathrm{~cm}^{-1}$ (blue) and another at $1587 \mathrm{~cm}^{-1}$ (red). Markers denote experimental data and solid lines show Gaussian fits. Global curve fitting was performed for all data shown in Figure 2.

Rather than reproduce the observed redshifts from empirical parameters, we investigated whether the experimental data could be simply fitted to two equilibrium species in relative ratios 
appropriate for labile metal-carboxylate bonds. Indeed, a global population analysis of the symmetric carboxylate stretch for HKUST-1 indicates that the temperature-dependent band can be accurately deconvoluted into a higher-energy component at $1596 \mathrm{~cm}^{-1}$ that decreases in intensity and a lower-energy component at $1587 \mathrm{~cm}^{-1}$ that increases in intensity with increased temperature (Figure 3). Importantly, the frequencies of these two components remains unchanged during the data fitting, as expected for a two-state model. Furthermore, this analysis corroborates the evidence in Figure $2 \mathrm{C}$ for a dominant species existing at either temperature extreme, but with nearly equal populations around $50{ }^{\circ} \mathrm{C}$. Scheme 2 illustrates this chemical scenario, where each component represents ensembles of metal carboxylate species with either long "loose" or short "tight" interactions, each integrated across shallow potential energy surfaces.

Scheme 2. Equilibrium between "tight" and "loose" ensembles of MOF metal-carboxylate populations existing near thermoneutral equilibrium. (Top) Conversion of MOF metal-linker bonds between two ensemble-averaged states. (Bottom) Temperature-dependent free energies $(\Delta G)$ and relative population according to equilibrium constants derived from experimental data.
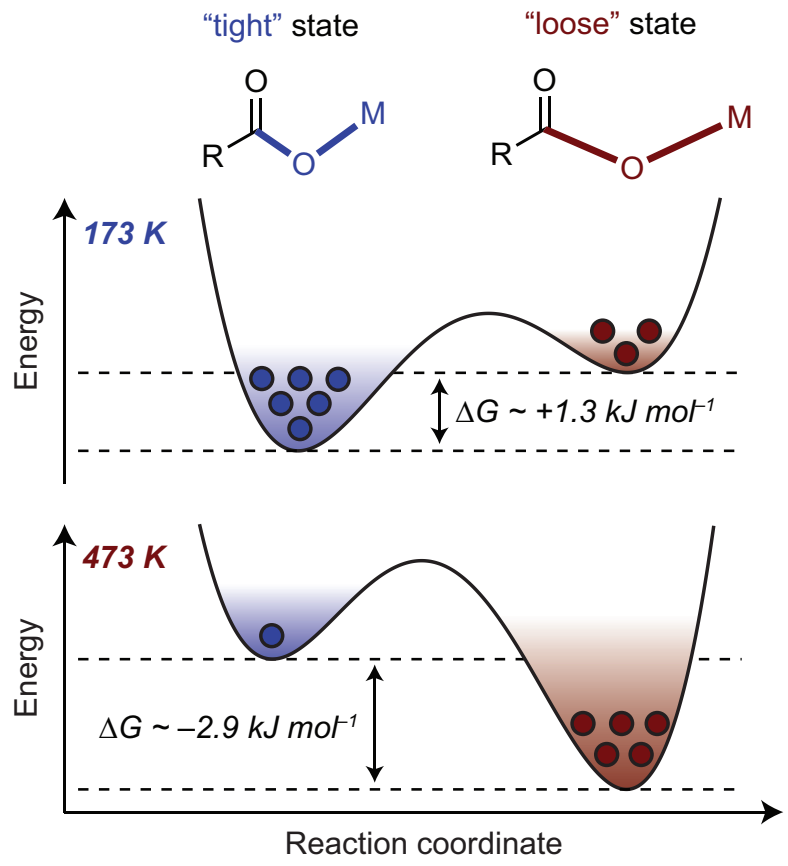


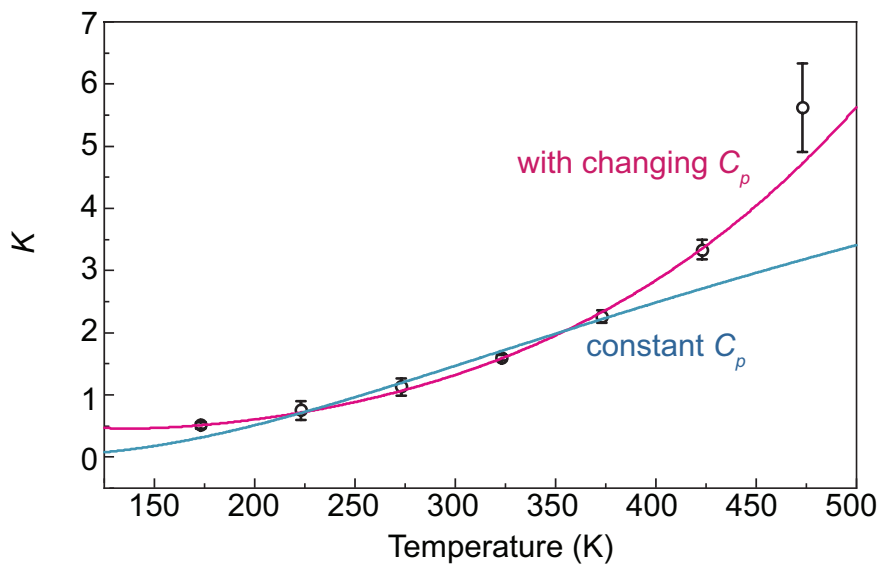

Figure 4: van't Hoff analysis of MOF metal-linker dynamic equilibrium. Inverse stability constants $K$ ([loose]/[tight]) were determined as the ratio of integrated spectral components determined by global curve fitting of the HKUST-1 asymmetric stretch. Data points represent average values from three temperature ramp cycles, with error bars denoting standard deviations. Data were fit to a standard van't Hoff equation assuming constant material specific heat (blue) and an equation with changing specific heat (pink).

To examine whether this spectral deconvolution supports the existence of dynamic metalcarboxylate bonds, we determined stability constants from the integrated relative ratios of both components at each temperature. As $K_{f}$ relates bound-to-unbound ratios by convention, Figure 4, instead, plots $K$ ([loose]/[tight] $)$ versus temperature for convenience because the lower-energy $1587 \mathrm{~cm}^{-1}$ feature associated with the loose state emerges with increased temperature. Importantly, the room temperature $\log K$ value of $\sim 0.6$ corresponds well to the reported values of molecular metal-carboxylate complexes, which range from $\sim 0.1$ to 1.5 for common MOF metal ions. ${ }^{37}$ Such small $K$ values imply a surprising degree of MOF metal-linker lability, in sharp contrast with the common conception of MOFs as static entities.

For further thermodynamic insight into the MOF metal-linker equilibrium, we performed van't Hoff analyses of the $K_{s p}{ }^{-1}$ versus temperature data. Figure 4 includes fits from a conventional analysis $(K=\exp [-(\Delta H-T \Delta S / R T)])$ and another $\left(K=\exp \left[-\left(\Delta H+\Delta C_{P}(T-298.15 \mathrm{~K})-T(\Delta S+\right.\right.\right.$ $\left.\left.\left.\left.\left.\Delta C_{P} \ln (T / 298.15 \mathrm{~K})\right)\right) / R T\right)\right]\right)$ that accounts for a changing material specific heat $\left(\Delta C_{P}\right)$, as has been 
applied previously to equilibrium mixtures of dynamic structures,${ }^{67}$ such as H-bonding networks and phase change materials. Interestingly, accounting for $\Delta C_{P}$ afforded a far superior fit to the data, lending further support for the red-shifting carboxylate stretches being indicators of lattice dynamical motion. This fitting procedure afforded values of $\Delta H=5.9 \pm 0.2 \mathrm{~kJ} \mathrm{~mol}^{-1}, \Delta S=22.0 \pm$ $0.6 \mathrm{~J} \mathrm{~mol}^{-1} \mathrm{~K}^{-1}$, and $\Delta C_{P}=37 \pm 4 \mathrm{~J} \mathrm{~mol}^{-1} \mathrm{~K}^{-1}$, suggesting this process involves a slight endothermic barrier overcome by increases in entropy, as expected for a thermally activated bond disruption process near equilibrium. A changing specific heat further supports the explanation that the redshifting spectra arise from soft mode behavior, as material specific heats become infinite near the critical temperatures of phase changes.

Redshifting MOF carboxylate stretches resemble lattice dynamical soft modes observed at temperatures near the phase transition critical temperatures $\left(T_{c}\right)$ for numerous materials, such as ferroelectric perovskites and ZIFs. ${ }^{5,6,68,69}$ Although described by different physical models, both involve large-amplitude vibrations driving structural phase changes. According to soft mode theory, the frequency of these critical vibrations redshifts and then vanishes as the material assumes a new structure with unique normal modes. In the data presented here, we attribute the redshift of the carboxylate modes to the gradual transition of a crystalline form into an amorphous structure with weaker metal-carboxylate bonds and a new corresponding set of normal modes. Interestingly, these data resemble the spectral evidence of melting behavior in ZIFs, and yet melting has yet to be observed in carboxylate MOFs. Computational simulations suggest, however, that metal-carboxylate soft modes drive breathing behavior in MOFs,${ }^{70}$ although direct evidence remains an outstanding challenge. These data, therefore, suggest that carboxylate-based MOFs undergo structural distortions associated with melting transitions, but decompose before reaching the melting point. A key difference that may explain the absence of melting carboxylate MOFs is 
that whereas ZIFs include individual metal ions, carboxylate MOFs often contain multi-metallic metal nodes. Upon dissociation from linkers, these complex inorganic fragments may recombine with nearby dangling linker molecules into any numerous possible polymorphs and amorphous decomposition products, rather than remain in a melted phase resembling the original crystalline lattice. While offering evidence for the existence for soft modes invoked to describe MOF liquids, these data provide mechanistic justification of other MOF phenomena requiring dissociation of MOF metal-linker bonds, most notably single-crystal-to-single-crystal post-synthetic modification (cation exchange and ligand exchange), ${ }^{47,52,53,71}$ the ability of MOFs to encapsulate molecules larger than their pore apertures, ${ }^{72}$ and the negative thermal expansion of MOFs. ${ }^{54-57,73-75}$

\section{Conclusion}

VT-DRIFTS provides strong evidence for dynamic metal-linker bonding in carboxylatebased MOFs. Redshifts to carboxylate vibrational modes can be modeled accurately as equilibrium mixtures of tight and loose metal-linker species, producing $K$ values remarkably consistent with the well-documented lability of molecular analogs. Additionally, carboxylate stretches serve as convenient spectroscopic alternatives to metal-linker vibrational modes for studying structural dynamics that have eluded recent investigations into liquid MOFs and MOF glasses. Taken together, this previously unobserved evidence for MOF metal-linker dynamics challenges the fundamental perception of MOFs as static structures, offering mechanistic justification for important MOF phenomena and a powerful tool for leveraging MOF structural dynamics in the design of next-generation adaptable materials.

\section{Associated Content}

Supporting Information: Syntheses, experimental details, supplementary figures and tables, additional VT-DRIFTS and DFT data. 


\section{Acknowledgements}

We gratefully acknowledge the University of Oregon for startup funds, and thank C. E. Bein and Profs. S. W. Boettcher, C. R. Wade, and D. C. Johnson for insightful conversations. This work made use of the CAMCOR facility of the Lorry I. Lokey Laboratories at the University of Oregon to perform VT-DRIFTS experiments. This material is based upon work supported by the National Science Foundation through the Division of Materials Research under grant no. DMR-1956403. We also acknowledge the continued support from the Extreme Science and Engineering Discovery Environment (XSEDE), which is supported by the National Science Foundation [ACI-1548562] and the PICS Coeus High Performance Computer, which is supported by the National Science Foundation [1624776].

\section{References}

(1) Famprikis, T.; Canepa, P.; Dawson, J. A.; Islam, M. S.; Masquelier, C. Fundamentals of Inorganic Solid-State Electrolytes for Batteries. Nat. Mater. 2019, 18 (12), 1278-1291.

(2) Sachs, C.; Hildebrand, M.; Volkening, S.; Wintterlin, J.; Ertl, G. Spatiotemporal SelfOrganization in a Surface Reaction: From the Atomic to the Mesoscopic Scale. Science 2001, 293 (5535), 1635-1638.

(3) Scott, J. F. Soft-Mode Spectroscopy: Experimental Studies of Structural Phase Transitions. Rev. Mod. Phys. 1974, 46 (1), 83-128.

(4) Cochran, W. Crystal Stability and the Theory of Ferroelectricity. Adv. Phys. 1960, 9 (36), 387-423.

(5) Dunnett, K.; Narayan, A.; Spaldin, N. A.; Balatsky, A. V. Strain and Ferroelectric SoftMode Induced Superconductivity in Strontium Titanate. Phys. Rev. B 2018, 97 (14), 1-8.

(6) Miyata, K.; Zhu, X. Y. Riddles in Perovskite Research: Ferroelectric Large Polarons. Nat. Mater. 2018, 17 (May), 379-381.

(7) Jiang, M. P.; Trigo, M.; Savic, I.; Fahy, S.; Murray, D.; Bray, C.; Clark, J.; Henighan, T.; Kozina, M.; Chollet, M.; et al. The Origin of Incipient Ferroelectricity in Lead Telluride. Nat. Commun. 2016, 7, 1-9.

(8) O'Hara, A.; Demkov, A. A. Nature of the Metal-Insulator Transition in $\mathrm{NbO}_{2}$. Phys. Rev. B - Condens. Matter Mater. Phys. 2015, 91 (9), 1-6.

(9) Wang, Z.; Rhodes, D. A.; Watanabe, K.; Taniguchi, T.; Hone, J. C.; Shan, J.; Mak, K. F. Evidence of High-Temperature Exciton Condensation in Two-Dimensional Atomic Double Layers. Nature 2019, 574 (7776), 76-80.

(10) Kogar, A.; Rak, M. S.; Vig, S.; Husain, A. A.; Flicker, F.; Joe, Y. Il; Venema, L.; MacDougall, G. J.; Chiang, T. C.; Fradkin, E.; et al. Signatures of Exciton Condensation in a Transition Metal Dichalcogenide. Science 2017, 358 (6368), 1314-1317. 
(11) Shuvaev, A. M.; Hemberger, J.; Niermann, D.; Schrettle, F.; Loidl, A.; Ivanov, V. Y.; Travkin, V. D.; Mukhin, A. A.; Pimenov, A. Soft-Mode Behavior of Electromagnons in Multiferroic Manganite. Phys. Rev. B - Condens. Matter Mater. Phys. 2010, 82 (17), 1-5.

(12) Cowley, R. A. Soft Modes and Structural Phase Transitions. Integr. Ferroelectr. 2012, 133 (1), 109-117.

(13) Fleury, P. A. The Effects of Soft Modes on the Structure and Properties of Materials. Annu. Rev. Mater. Sci. 1976, 6 (1), 157-180.

(14) Gaillac, R.; Pullumbi, P.; Beyer, K. A.; Chapman, K.; Keen, D. A.; Bennett, T. D.; Coudert, F. X. Liquid Metal-Organic Frameworks. Nat. Mater. 2017, 16 (11), 1149-1155.

(15) Costa Gomes, M.; Pison, L.; Červinka, C.; Padua, A. Porous Ionic Liquids or Liquid Metal-Organic Frameworks? Angew. Chem. Int. Ed. 2018, 6, 11909-11912.

(16) Longley, L.; Collins, S. M.; Li, S.; Smales, G. J.; Erucar, I.; Qiao, A.; Hou, J.; Doherty, C. M.; Thornton, A. W.; Hill, A. J.; et al. Flux Melting of Metal-Organic Frameworks. Chem. Sci. 2019, 10 (12), 3592-3601.

(17) Bumstead, A. M.; Ríos Gómez, M. L.; Thorne, M. F.; Sapnik, A. F.; Longley, L.; Tuffnell, J. M.; Keeble, D. S.; Keen, D. A.; Bennett, T. D. Investigating the Melting Behaviour of Polymorphic Zeolitic Imidazolate Frameworks. CrystEngComm 2020, 22 (21), 36273637.

(18) Tuffnell, J. M.; Ashling, C. W.; Hou, J.; Li, S.; Longley, L.; Ríos Gómez, M. L.; Bennett, T. D. Novel Metal-Organic Framework Materials: Blends, Liquids, Glasses and CrystalGlass Composites. Chem. Commun. 2019, 55 (60), 8705-8715.

(19) Bennett, T. D.; Horike, S. Liquid, Glass and Amorphous Solid States of Coordination Polymers and Metal-Organic Frameworks. Nat. Rev. Mater. 2018, 3 (11), 431-440.

(20) Ogawa, T.; Takahashi, K.; Nagarkar, S. S.; Ohara, K.; Hong, Y.; Nishiyama, Y.; Horike, S. Coordination Polymer Glass from a Protic Ionic Liquid: Proton Conductivity and Mechanical Properties as an Electrolyte. Chem. Sci. 2020, 11 (20), 5175-5181.

(21) Horike, S.; Nagarkar, S. S.; Ogawa, T.; Kitagawa, S. A New Dimension for Coordination Polymers and Metal-Organic Frameworks: Towards Functional Glasses and Liquids. Angew. Chem. Int. Ed. 2020, 59 (17), 6652-6664.

(22) Chen, W.; Horike, S.; Umeyama, D.; Ogiwara, N.; Itakura, T.; Tassel, C.; Goto, Y.; Kageyama, H.; Kitagawa, S. Glass Formation of a Coordination Polymer Crystal for Enhanced Proton Conductivity and Material Flexibility. Angew. Chem. Int. Ed. 2016, 55 (17), 5195-5200.

(23) Zhou, C.; Longley, L.; Krajnc, A.; Smales, G. J.; Qiao, A.; Erucar, I.; Doherty, C. M.; Thornton, A. W.; Hill, A. J.; Ashling, C. W.; et al. Metal-Organic Framework Glasses with Permanent Accessible Porosity. Nat. Commun. 2018, 9 (1), 1-9.

(24) Hou, J.; Ríos Gómez, M. L.; Krajnc, A.; McCaul, A.; Li, S.; Bumstead, A. M.; Sapnik, A. F.; Deng, Z.; Lin, R.; Chater, P. A.; et al. Halogenated Metal-Organic Framework Glasses and Liquids. J. Am. Chem. Soc. 2020, 142 (8), 3880-3890.

(25) Umeyama, D.; Funnell, N. P.; Cliffe, M. J.; Hill, J. A.; Goodwin, A. L.; Hijikata, Y.; Itakura, T.; Okubo, T.; Horike, S.; Kitagawa, S. Glass Formation via Structural Fragmentation of a 2D Coordination Network. Chem. Commun. 2015, 51 (64), 1272812731.

(26) Hou, J.; Ashling, C. W.; Collins, S. M.; Krajnc, A.; Zhou, C.; Longley, L.; Johnstone, D. N.; Chater, P. A.; Li, S.; Coulet, M. V.; et al. Metal-Organic Framework Crystal-Glass Composites. Nat. Commun. 2019, 10 (1), 1-10. 
(27) Brozek, C. K.; Michaelis, V. K.; Ong, T.-C.; Bellarosa, L.; López, N.; Griffin, R. G.; Dincă, M. Dynamic DMF Binding in MOF-5 Enables the Formation of Metastable Cobalt-Substituted MOF-5 Analogues. ACS Cent. Sci. 2015, 1 (5), 252-260.

(28) Krylov, A.; Vtyurin, A.; Petkov, P.; Senkovska, I.; Maliuta, M.; Bon, V.; Heine, T.; Kaskel, S.; Slyusareva, E. Raman Spectroscopy Studies of the Terahertz Vibrational Modes of a DUT-8 (Ni) Metal-Organic Framework. Phys. Chem. Chem. Phys. 2017, 19 (47), 32099-32104.

(29) Hoffman, A. E. J.; Vanduyfhuys, L.; Nevjestić, I.; Wieme, J.; Rogge, S. M. J.; Depauw, H.; Van Der Voort, P.; Vrielinck, H.; Van Speybroeck, V. Elucidating the Vibrational Fingerprint of the Flexible Metal-Organic Framework MIL-53(Al) Using a Combined Experimental/Computational Approach. J. Phys. Chem. C 2018, 122 (5), 2734-2746.

(30) Rao, Y. L.; Chortos, A.; Pfattner, R.; Lissel, F.; Chiu, Y. C.; Feig, V.; Xu, J.; Kurosawa, T.; Gu, X.; Wang, C.; et al. Stretchable Self-Healing Polymeric Dielectrics Cross-Linked through Metal-Ligand Coordination. J. Am. Chem. Soc. 2016, 138 (18), 6020-6027.

(31) Williams, K. A.; Boydston, A. J.; Bielawski, C. W. Main-Chain Organometallic Polymers: Synthetic Strategies, Applications, and Perspectives. Chem. Soc. Rev. 2007, 36 (5), 729.

(32) Norris, B. C.; Bielawski, C. W. Structurally Dynamic Materials Based on Bis(NHeterocyclic Carbene)s and Bis(Isothiocyanate)s: Toward Reversible, Conjugated Polymers. Macromolecules 2010, 43 (8), 3591-3593.

(33) Williams, K. A.; Dreyer, D. R.; Bielawski, C. W. The Underlying Chemistry of SelfHealing Materials. MRS Bull. 2008, 33 (08), 759-765.

(34) Winter, A.; Schubert, U. S. Synthesis and Characterization of Metallo-Supramolecular Polymers. Chem. Soc. Rev. 2016, 45 (19), 5311-5357.

(35) Dobrawa, R.; Würthner, F. Metallosupramolecular Approach toward Functional Coordination Polymers. J. Polym. Sci. Part A Polym. Chem. 2005, 43 (21), 4981-4995.

(36) Lewis, A. K. de K.; Caddick, S.; Cloke, F. G. N.; Billingham, N. C.; Hitchcock, P. B.; Leonard, J. Synthetic, Structural, and Mechanistic Studies on the Oxidative Addition of Aromatic Chlorides to a Palladium (N-Heterocyclic Carbene) Complex: Relevance to Catalytic Amination. J. Am. Chem. Soc. 2003, 125 (33), 10066-10073.

(37) Bunting, J. W.; Thong, K. M. Stability Constants for Some 1:1 Metal-Carboxylate Complexes. Can. J. Chem. 1970, 48 (11), 1654-1656.

(38) Grosch, J. S.; Paesani, F. Molecular-Level Characterization of the Breathing Behavior of the Jungle-Gym-Type DMOF-1 Metal-Organic Framework. J. Am. Chem. Soc. 2012, 134 (9), 4207-4215.

(39) Yot, P. G.; Ma, Q.; Haines, J.; Yang, Q.; Ghoufi, A.; Devic, T.; Serre, C.; Dmitriev, V.; Férey, G.; Zhong, C.; et al. Large Breathing of the MOF MIL-47(VIV) under Mechanical Pressure: A Joint Experimental-Modelling Exploration. Chem. Sci. 2012, 3 (4), 11001104.

(40) Ghoufi, A.; Benhamed, K.; Boukli-Hacene, L.; Maurin, G. Electrically Induced Breathing of the MIL-53(Cr) Metal-Organic Framework. ACS Cent. Sci. 2017, 3 (5), 394-398.

(41) Parent, L. R.; Pham, C. H.; Patterson, J. P.; Denny, M. S.; Cohen, S. M.; Gianneschi, N. C.; Paesani, F. Pore Breathing of Metal-Organic Frameworks by Environmental Transmission Electron Microscopy. J. Am. Chem. Soc. 2017, 139 (40), 13973-13976.

(42) Nishida, J.; Tamimi, A.; Fei, H.; Pullen, S.; Ott, S.; Cohen, S. M.; Fayer, M. D. Structural Dynamics inside a Functionalized Metal-Organic Framework Probed by Ultrafast 2D IR Spectroscopy. Proc. Natl. Acad. Sci. U. S. A. 2014, 111 (52), 18442-18447. 
(43) Mao, V. Y.; Milner, P. J.; Lee, J.-H.; Forse, A. C.; Kim, E. J.; Siegelman, R. L.; McGuirk, C. M.; Porter-Zasada, L. B.; Neaton, J. B.; Reimer, J. A.; et al. Cooperative Carbon Dioxide Adsorption in Alcoholamine- and Alkoxyalkylamine-Functionalized MetalOrganic Frameworks. Angew. Chem. Int. Ed. 2020, 2-12.

(44) McDonald, T. M.; Mason, J. A.; Kong, X.; Bloch, E. D.; Gygi, D.; Dani, A.; Crocellà, V.; Giordanino, F.; Odoh, S. O.; Drisdell, W. S.; et al. Cooperative Insertion of $\mathrm{CO}_{2}$ in Diamine-Appended Metal-Organic Frameworks. Nature 2015, 519 (7543), 303-308.

(45) Kong, X.; Scott, E.; Ding, W.; Mason, J. A.; Long, J. R.; Reimer, J. A. CO 2 Dynamics in a Metal-Organic Framework with Open Metal Sites. J. Am. Chem. Soc. 2012, 134 (35), 14341-14344.

(46) Brozek, C. K.; Ozarowski, A.; Stoian, S. A.; Dincă, M. Dynamic Structural Flexibility of Fe-MOF-5 Evidenced by ${ }^{57}$ Fe Mössbauer Spectroscopy. Inorg. Chem. Front. 2017, 4 (5), $782-788$.

(47) Brozek, C. K.; Dincă, M. Cation Exchange at the Secondary Building Units of MetalOrganic Frameworks. Chem. Soc. Rev. 2014, 43 (16), 5456-5467.

(48) Brozek, C. K.; Dincă, M. Lattice-Imposed Geometry in Metal-Organic Frameworks: Lacunary Zn4O Clusters in MOF-5 Serve as Tripodal Chelating Ligands for $\mathrm{Ni}^{2+}$. Chem. Sci. 2012, 3 (6), 2110.

(49) Brozek, C. K.; Bellarosa, L.; Soejima, T.; Clark, T. V.; Lõpez, N.; Dincă, M. SolventDependent Cation Exchange in Metal-Organic Frameworks. Chem.-Eur. J. 2014, 20, 6871.

(50) Brozek, C. K.; Dincă, M. Thermodynamic Parameters of Cation Exchange in MOF-5 and MFU-41. Chem. Commun. 2015, 51 (59), 11780-11782.

(51) Takaishi, S.; DeMarco, E. J.; Pellin, M. J.; Farha, O. K.; Hupp, J. T. Solvent-Assisted Linker Exchange (SALE) and Post-Assembly Metallation in Porphyrinic Metal-Organic Framework Materials. Chem. Sci. 2013, 4 (4), 1509.

(52) Kim, M.; Cahill, J. F.; Su, Y.; Prather, K. A.; Cohen, S. M. Postsynthetic Ligand Exchange as a Route to Functionalization of "inert" Metal-Organic Frameworks. Chem. Sci. 2012, 3 (1), 126-130.

(53) Cohen, S. M. The Postsynthetic Renaissance in Porous Solids. J. Am. Chem. Soc. 2017, 139 (8), 2855-2863.

(54) Wu, Y.; Kobayashi, A.; Halder, G. J.; Peterson, V. K.; Chapman, K. W.; Lock, N.; Southon, P. D.; Kepert, C. J. Negative Thermal Expansion in the Metal-Organic Framework Material $\mathrm{Cu}_{3}(1,3,5$-Benzenetricarboxylate)2. Angew. Chem. Int. Ed. 2008, 47 (46), 8929-8932.

(55) Lock, N.; Christensen, M.; Kepert, C. J.; Iversen, B. B. Effect of Gas Pressure on Negative Thermal Expansion in MOF-5. Chem. Commun. 2013, 49 (8), 789-791.

(56) Zhou, W.; Wu, H.; Yildirim, T.; Simpson, J. R.; Walker, A. R. H. Origin of the Exceptional Negative Thermal Expansion in Metal-Organic Framework-5 Zn ${ }_{4} \mathrm{O}(1,4-$ Benzenedicarboxylate) 3. Phys. Rev. B - Condens. Matter Mater. Phys. 2008, 78 (5), 1-5.

(57) Schneider, C.; Bodesheim, D.; Ehrenreich, M. G.; Crocella, V.; Mink, J.; Fischer, R. A.; Butler, K. T.; Kieslich, G. Tuning the Negative Thermal Expansion Behavior of the Metal- Organic Framework $\mathrm{Cu}_{3} \mathrm{BTC}_{2}$ by Retrofitting. J. Am. Chem. Soc. 2019, 141 (26), 10504-10509.

(58) Redfern, L. R.; Ducamp, M.; Wasson, M. C.; Robison, L.; Son, F. A.; Coudert, F.-X.; Farha, O. K. Isolating the Role of the Node-Linker Bond in the Compression of UiO-66 
Metal-Organic Frameworks. Chem. Mater. 2020.

(59) Leus, K.; Muylaert, I.; Vandichel, M.; Marin, G. B.; Waroquier, M.; Van Speybroeck, V.; Van der Voort, P. The Remarkable Catalytic Activity of the Saturated Metal Organic Framework V-MIL-47 in the Cyclohexene Oxidation. Chem. Commun. 2010, 46 (28), 5085.

(60) Bode, S.; Zedler, L.; Schacher, F. H.; Dietzek, B.; Schmitt, M.; Popp, J.; Hager, M. D.; Schubert, U. S. Self-Healing Polymer Coatings Based on Crosslinked Metallosupramolecular Copolymers. Adv. Mater. 2013, 25 (11), 1634-1638.

(61) Shen, Y. C.; Upadhya, P. C.; Linfield, E. H.; Davies, A. G. Temperature-Dependent LowFrequency Vibrational Spectra of Purine and Adenine. Appl. Phys. Lett. 2003, 82 (14), 2350-2352.

(62) Chakravarty, C.; Debenedetti, P. G.; Stillinger, F. H. Lindemann Measures for the SolidLiquid Phase Transition. J. Chem. Phys. 2007, 126 (20).

(63) Tiana, D.; Hendon, C. H.; Walsh, A. Ligand Design for Long-Range Magnetic Order in Metal-Organic Frameworks. Chem. Commun. 2014, 50 (90), 13990-13993.

(64) Xu, B.; Poduska, K. M. Linking Crystal Structure with Temperature-Sensitive Vibrational Modes in Calcium Carbonate Minerals. Phys. Chem. Chem. Phys. 2014, 16 (33), 1763417639.

(65) Balan, E.; Delattre, S.; Roche, D.; Segalen, L.; Morin, G.; Guillaumet, M.; Blanchard, M.; Lazzeri, M.; Brouder, C.; Salje, E. K. H. Line-Broadening Effects in the Powder Infrared Spectrum of Apatite. Phys. Chem. Miner. 2011, 38 (2), 111-122.

(66) Bien, C. E.; Chen, K. K.; Chien, S.-C.; Reiner, B. R.; Lin, L.-C.; Wade, C. R.; Ho, W. S. W. Bioinspired Metal-Organic Framework for Trace $\mathrm{CO}_{2}$ Capture. J. Am. Chem. Soc. 2018, 140 (40), 12662-12666.

(67) Huang, C. Y.; Wang, T.; Gai, F. Temperature Dependence of the CN Stretching Vibration of a Nitrile-Derivatized Phenylalanine in Water. Chem. Phys. Lett. 2003, 371 (5-6), 731738.

(68) Sirenko, A. A.; Clark, A. M.; Hao, J.; Si, W.; Xi, X. X. Soft-Mode Hardening in $\mathrm{SrTiO}_{3}$ Thin Films. Nature 2000, 9, 373-376.

(69) Zhou, J. J.; Hellman, O.; Bernardi, M. Electron-Phonon Scattering in the Presence of Soft Modes and Electron Mobility in $\mathrm{SrTiO}_{3}$ Perovskite from First Principles. Phys. Rev. Lett. 2018, 121 (22), 226603.

(70) Hoffman, A. E. J.; Wieme, J.; Rogge, S. M. J.; Vanduyfhuys, L.; Van Speybroeck, V. The Impact of Lattice Vibrations on the Macroscopic Breathing Behavior of MIL-53(Al). Zeitschrift für Krist. - Cryst. Mater. 2019, 234 (7-8), 529-545.

(71) Liu, L.; Li, L.; Ziebel, M. E.; Harris, T. D. Metal-Diamidobenzoquinone Frameworks via Post-Synthetic Linker Exchange. J. Am. Chem. Soc. 2020, 142 (10), 4705-4713.

(72) Morabito, J. V.; Chou, L. Y.; Li, Z.; Manna, C. M.; Petroff, C. A.; Kyada, R. J.; Palomba, J. M.; Byers, J. A.; Tsung, C. K. Molecular Encapsulation beyond the Aperture Size Limit through Dissociative Linker Exchange in Metal-Organic Framework Crystals. J. Am. Chem. Soc. 2014, 136 (36), 12540-12543.

(73) Balestra, S. R. G.; Bueno-Perez, R.; Hamad, S.; Dubbeldam, D.; Ruiz-Salvador, A. R.; Calero, S. Controlling Thermal Expansion: A Metal-Organic Frameworks Route. Chem. Mater. 2016, 28 (22), 8296-8304.

(74) Lock, N.; Wu, Y.; Christensen, M.; Cameron, L. J.; Peterson, V. K.; Bridgeman, A. J.; Kepert, C. J.; Iversen, B. B. Elucidating Negative Thermal Expansion in MOF-5. J. Phys. 
Chem. C 2010, 114 (39), 16181-16186.

(75) Rimmer, L. H. N.; Dove, M. T.; Goodwin, A. L.; Palmer, D. C. Acoustic Phonons and Negative Thermal Expansion in MOF-5. Phys. Chem. Chem. Phys. 2014, 16 (39), 21144 21152. 